\title{
Radon-Nikodým compact spaces of low weight and Banach spaces
}

\author{
by
}

\author{
Antonio Avilés (Murcia)
}

\begin{abstract}
We prove that a continuous image of a Radon-Nikodým compact of weight less than $\mathbf{b}$ is Radon-Nikodým compact. As a Banach space counterpart, subspaces of Asplund generated Banach spaces of density character less than $\mathbf{b}$ are Asplund generated. In this case, in addition, there exists a subspace of an Asplund generated space which is not Asplund generated and which has density character exactly $\mathbf{b}$.
\end{abstract}

The concept of Radon-Nikodým compact, due to Reynov [13], has its origin in Banach space theory, and it is defined as a topological space which is homeomorphic to a weak* compact subset of the dual of an Asplund space, that is, a dual Banach space with the Radon-Nikodým property (topological spaces will be here assumed to be Hausdorff). In [10], the following characterization of this class is given:

TheOREM 1. A compact space $K$ is Radon-Nikodym compact if and only if there is a lower semicontinuous metric $d$ on $K$ which fragments $K$.

Recall that a map $f: X \times X \rightarrow \mathbb{R}$ on a topological space $X$ is said to fragment $X$ if for each (closed) subset $L$ of $X$ and each $\varepsilon>0$ there is a nonempty relatively open subset $U$ of $L$ of $f$-diameter less than $\varepsilon$, i.e. $\sup \{f(x, y): x, y \in U\}<\varepsilon$. Also, a map $g: Y \rightarrow \mathbb{R}$ from a topological space to the real line is lower semicontinuous if $\{y: g(y) \leq r\}$ is closed in $Y$ for every real number $r$.

It is an open problem whether a continuous image of a Radon-Nikodým compact is Radon-Nikodým. Arvanitakis [2] has taken the following approach to this problem: if $K$ is a Radon-Nikodým compact and $\pi: K \rightarrow L$ is a continuous surjection, then we have a lower semicontinuous fragmenting

2000 Mathematics Subject Classification: Primary 46B26; Secondary 46B22, 46B50, $54 \mathrm{G} 99$.

Key words and phrases: Radon-Nikodým compact, quasi-Radon-Nikodým compact, countably lower fragmentable compact, Asplund generated space, weakly $\mathcal{K}$-analytic space, weakly compactly generated space, cardinal b, Martin's axiom.

Author supported by FPU grant of SEEU-MECD of Spain. 
metric $d$ on $K$, and if we want to prove that $L$ is Radon-Nikodým compact, we should find such a metric on $L$. A natural candidate is

$$
d_{1}(x, y)=d\left(\pi^{-1}(x), \pi^{-1}(y)\right)=\inf \{d(t, s): \pi(t)=x, \pi(s)=y\} .
$$

The map $d_{1}$ is lower semicontinuous and fragments $L$ and it is a quasimetric, that is, it is symmetric and vanishes only if $x=y$. But it is not a metric because, in general, it fails the triangle inequality. Consequently, Arvanitakis [2] introduced the following concept:

Definition 2. A compact space $L$ is said to be quasi-Radon-Nikodým if there exists a lower semicontinuous quasi-metric which fragments $L$.

The class of quasi-Radon-Nikodým compacta is closed under continuous images but it is unknown whether it is the same class as that of RadonNikodým compacta or even the class of their continuous images. At least two other superclasses of continuous images of Radon-Nikodým compacta appear in the literature. Reznichenko [1, p. 104] defined a compact space $L$ to be strongly fragmentable if there is a metric $d$ which fragments $L$ such that each pair of different points of $L$ have disjoint neighbourhoods at a positive $d$-distance. It has been noted by Namioka [11] that the classes of quasi-Radon-Nikodým and strongly fragmentable compacta are equal. Another superclass of continuous images of Radon-Nikodým compacta, called countably lower fragmentable compacta, was introduced by Fabian, Heisler and Matoušková [6]. In Section 3, we recall their definition and we prove that this class is equal to the other two.

The main result in Section 1 is the following:

THEOREM 3. If $K$ is a quasi-Radon-Nikodým compact space of weight less than $\mathbf{b}$, then $K$ is Radon-Nikodým compact.

The weight of a topological space is the least cardinality of a base for its topology. We also recall the definition of the cardinal b. In the set $\mathbb{N}^{\mathbb{N}}$ we consider the order relation given by $\sigma \leq \tau$ if $\sigma_{n} \leq \tau_{n}$ for all $n \in \mathbb{N}$. The cardinal $\mathbf{b}$ is the least cardinality of a subset of $\mathbb{N}^{\mathbb{N}}$ which is not $\sigma$ bounded for this order (a set is $\sigma$-bounded if it is a countable union of bounded subsets). It is consistent that $\mathbf{b}>\omega_{1}$. In fact, Martin's axiom and the negation of the continuum hypothesis imply that $\mathbf{c}=\mathbf{b}>\omega_{1}$ (cf. [7, $11 \mathrm{D}$ and $14 \mathrm{~B}]$ ). It is also possible that $\mathbf{c}>\mathbf{b}>\omega_{1}$ (cf. [4, Section 5]). On the other hand, the cardinal $\mathbf{d}$ is the least cardinality of a cofinal subset of $\left(\mathbb{N}^{\mathbb{N}}, \leq\right)$, that is, a set $A$ such that for each $\sigma \in \mathbb{N}^{\mathbb{N}}$ there is some $\tau \in A$ such that $\sigma \leq \tau$. In a sense, the following proposition puts a rough bound on the size of the class of quasi-Radon-Nikodým compacta with respect to Radon-Nikodým compacta. 
Proposition 4. Every quasi-Radon-Nikodým compact space embeds into a product of Radon-Nikodým compact spaces with at most $\mathbf{d}$ factors.

In Section 2 we discuss the Banach space counterpart to Theorem 3. A Banach space $V$ is Asplund generated, or GSG, if there is some Asplund space $V^{\prime}$ and a bounded linear operator $T: V^{\prime} \rightarrow V$ such that $T\left(V^{\prime}\right)$ is dense in $V$. Our main result for this class is the following:

THEOREM 5. Let $V$ be a Banach space of density character less than $\mathbf{b}$ and such that the dual unit ball $\left(B_{V^{*}}, w^{*}\right)$ is quasi-Radon-Nikodým compact. Then $V$ is Asplund generated.

The density character of a Banach space is the least cardinal of a normdense subset, and it equals the weight of its dual unit ball in the weak* topology.

Examples constructed by Rosenthal [14] and Argyros [5, Section 1.6] show that there exist Banach spaces which are subspaces of Asplund generated spaces but which are not Asplund generated. However, since the dual unit ball of a subspace of an Asplund generated space is a continuous image of a Radon-Nikodým compact [5, Theorem 1.5.6], we have the following corollary to Theorem 5:

Corollary 6. If a Banach space $V$ is a subspace of an Asplund generated space and the density character of $V$ is less than $\mathbf{b}$, then $V$ is Asplund generated.

Also, a Banach space is weakly compactly generated (WCG) if it is the closed linear span of a weakly compact subset. The same examples mentioned above show that this property is not inherited by subspaces. A Banach space $V$ is weakly compactly generated if and only if it is Asplund generated and its dual unit ball $\left(B_{V^{*}}, w^{*}\right)$ is Corson compact [12], [15]. Having Corson dual unit ball is a hereditary property since a continuous image of a Corson compact is Corson compact [8], hence:

COROLlary 7. If a Banach space $V$ is a subspace of a weakly compactly generated space and the density character of $V$ is less than $\mathbf{b}$, then $V$ is weakly compactly generated.

Corollary 7 can also be obtained from the following theorem, essentially due to Mercourakis [9]:

THEOREM 8. If a Banach space $V$ is weakly $\mathcal{K}$-analytic and the density character of $V$ is less than $\mathbf{b}$, then $V$ is weakly compactly generated.

The class of weakly $\mathcal{K}$-analytic spaces is larger than the class of subspaces of weakly compactly generated spaces. We recall its definition in Section 2. The result of Mercourakis [9, Theorem 3.13] is that, under Martin's axiom, 
weakly $\mathcal{K}$-analytic Banach spaces of density character less than $\mathbf{c}$ are weakly compactly generated, but his arguments prove in fact the more general Theorem 8 . We give a more elementary proof of this theorem, obtaining it as a consequence of a purely topological result: Any $\mathcal{K}$-analytic topological space of density character less than $\mathbf{b}$ contains a dense $\sigma$-compact subset. We also remark that it is not possible to generalize Theorem 8 to the class of weakly countably determined Banach spaces.

The cardinal $\mathbf{b}$ is best possible for Theorems 5 and 8 and their corollaries, as shown by slight modifications of the above mentioned example of Argyros [5, Section 1.6] and of the example of Talagrand [16] of a weakly $\mathcal{K}$-analytic Banach space which is not weakly compactly generated, so that we get examples of density character exactly $\mathbf{b}$.

For information about the cardinals $\mathbf{b}$ and $\mathbf{d}$ we refer to [4]. Concerning Banach spaces, our main reference is [5].

I want to express my gratitude to José Orihuela for valuable discussions and suggestions, and to Witold Marciszewski, from whom I learnt about the cardinals $\mathbf{b}$ and $\mathbf{d}$. I also thank Isaac Namioka and the referee for suggestions which have improved the final version of this article.

1. Quasi-Radon-Nikodým compacta of low weight. In this section, we characterize quasi-Radon-Nikodým compacta in terms of embeddings into cubes $[0,1]^{\Gamma}$, and from this we derive proofs of Theorem 3 and Proposition 4. Techniques of Arvanitakis [2] play an important role in this section, as well as the following theorem of Namioka [10]:

THEOREM 9. Let $K$ be a compact space. The following are equivalent:

(1) $K$ is Radon-Nikodým compact.

(2) There is an embedding $K \subset[0,1]^{\Gamma}$ such that $K$ is fragmented by the uniform metric $d(x, y)=\sup _{\gamma \in \Gamma}\left|x_{\gamma}-y_{\gamma}\right|$.

Let $\mathrm{P} \subset \mathbb{N}^{\mathbb{N}}$ be the set of all strictly increasing sequences of positive integers. Note that this is a cofinal subset of $\mathbb{N}^{\mathbb{N}}$. For each $\sigma \in \mathrm{P}$ we consider the lower semicontinuous nondecreasing function $h^{\sigma}:[0, \infty] \rightarrow \mathbb{R}$ given by

$$
h^{\sigma}(t)= \begin{cases}0 & \text { for } t=0 \\ 1 / \sigma_{n} & \text { whenever } 1 /(n+1)<t \leq 1 / n \\ 1 / \sigma_{1} & \text { whenever } t>1\end{cases}
$$

Also, if $f: X \times X \rightarrow \mathbb{R}$ is a map and $A, B \subset X$, we will use the notation $f(A, B)=\inf \{f(x, y): x \in A, y \in B\}$.

THEOREM 10. Let $K$ be a compact subset of the cube $[0,1]^{\Gamma}$. The following are equivalent: 
(1) $K$ is quasi-Radon-Nikodým compact.

(2) There is a map $\sigma: \Gamma \rightarrow \mathrm{P}$ such that $K$ is fragmented by

$$
f(x, y)=\sup _{\gamma \in \Gamma} h^{\sigma(\gamma)}\left(\left|x_{\gamma}-y_{\gamma}\right|\right),
$$

which is a lower semicontinuous quasi-metric.

Proof. Observe that $f$ in (2) is expressed as a supremum of lower semicontinuous functions, and therefore it is lower semicontinuous. Also, $f(x, y)$ $=0$ if and only if $h^{\sigma(\gamma)}\left(\left|x_{\gamma}-y_{\gamma}\right|\right)=0$ for all $\gamma \in \Gamma$ if and only if $\left|x_{\gamma}-y_{\gamma}\right|=0$ for all $\gamma \in \Gamma$. Hence, $f$ is indeed a lower semicontinuous quasi-metric and it is clear that (2) implies (1). Assume now that $K$ is quasi-Radon-Nikodým compact and let $g: K \times K \rightarrow[0,1]$ be a lower semicontinuous quasi-metric which fragments $K$. For $\gamma \in \Gamma$, we denote by $p_{\gamma}: K \rightarrow[0,1]$ the projection on the coordinate $\gamma, p_{\gamma}(x)=x_{\gamma}$, and we define a quasi-metric $g_{\gamma}$ on $[0,1]$ by the rule

$$
g_{\gamma}(t, s)= \begin{cases}g\left(p_{\gamma}^{-1}(t), p_{\gamma}^{-1}(s)\right) & \text { if } p_{\gamma}^{-1}(t) \text { and } p_{\gamma}^{-1}(s) \text { are nonempty } \\ 1 & \text { otherwise. }\end{cases}
$$

Note that $g_{\gamma}$ is lower semicontinuous because for $r<1$,

$$
\left\{(t, s): g_{\gamma}(t, s) \leq r\right\}=\bigcap_{r^{\prime}>r}\left(p_{\gamma} \times p_{\gamma}\right)\left\{(x, y) \in K^{2}: g(x, y) \leq r^{\prime}\right\}
$$

Observe also that if $x, y \in K$, then $g_{\gamma}\left(x_{\gamma}, y_{\gamma}\right)=g_{\gamma}\left(p_{\gamma}(x), p_{\gamma}(y)\right) \leq g(x, y)$. Hence, $K$ is fragmented by

$$
g^{\prime}(x, y)=\sup _{\gamma \in \Gamma} g_{\gamma}\left(x_{\gamma}, y_{\gamma}\right) \leq g(x, y) .
$$

The proof finishes by making use of the following lemma, where we put $g_{0}:=g_{\gamma}:$

Lemma 11. Let $g_{0}:[0,1] \times[0,1] \rightarrow[0,1]$ be a lower semicontinuous quasi-metric on $[0,1]$. Then there exists $\tau \in \mathrm{P}$ such that $h^{\tau}(|t-s|) \leq g_{0}(t, s)$ for all $t, s \in[0,1]$.

Proof. We define $\tau$ recursively. Suppose that we have defined $\tau_{1}, \ldots, \tau_{n}$ in such a way that if $|t-s|>1 /(n+1)$, then $h^{\tau}(|t-s|) \leq g_{0}(t, s)$. Let

$$
K_{m}=\left\{(t, s) \in[0,1] \times[0,1]:|t-s| \geq 1 /(n+2) \text { and } g_{0}(t, s) \leq 1 / m\right\} .
$$

Then $\left\{K_{m}\right\}_{m=1}^{\infty}$ is a decreasing sequence of compact subsets of $[0,1]^{2}$ with empty intersection. Hence, there is $m_{1}$ such that $K_{m}$ is empty for $m \geq m_{1}$. We define $\tau_{n+1}=\max \left\{m_{1}, \tau_{n}+1\right\}$.

Now, we state a lemma which is just a piece of the proof of [2, Proposition 3.2]. We include its proof for the sake of completeness.

Lemma 12. Let $K, L$ be compact spaces, let $f: K \times K \rightarrow \mathbb{R}$ be a symmetric map which fragments $K$ and $p: K \rightarrow L$ a continuous surjection. 
Then $L$ is fragmented by $g(x, y)=f\left(p^{-1}(x), p^{-1}(y)\right)$, and in particular, $L$ is fragmented by any $g^{\prime}$ with $g^{\prime} \leq g$.

Proof. Let $M$ be a closed subset of $L$ and $\varepsilon>0$. By Zorn's lemma a set $N \subset K$ can be found such that $p: N \rightarrow M$ is onto and irreducible (that is, for every $N^{\prime} \subset N$ closed, $p: N^{\prime} \rightarrow M$ is not onto). We find a relatively open subset $U \subset N$ of $f$-diameter less than $\varepsilon$. By irreducibility, $p(U)$ has nonempty relative interior in $M$. This interior is a nonempty relative open subset of $M$ of $g$-diameter less than $\varepsilon$.

In what follows, we use the following notation: If $A \subset \Gamma$ are sets, $d_{A}$ stands for the pseudometric in $[0,1]^{\Gamma}$ given by $d_{A}(x, y)=\sup _{\gamma \in A}\left|x_{\gamma}-y_{\gamma}\right|$.

LEMMA 13. Let $K$ be a compact subset of the cube $[0,1]^{\Gamma}$ and let $\sigma$ : $\Gamma \rightarrow \mathrm{P}$ be a map such that the quasi-metric

$$
f(x, y)=\sup _{\gamma \in \Gamma} h^{\sigma(\gamma)}\left(\left|x_{\gamma}-y_{\gamma}\right|\right)
$$

fragments $K$ and $\sigma(\Gamma)$ is a $\sigma$-bounded subset of $\mathbb{N}^{\mathbb{N}}$. Then $K$ is RadonNikodým compact. In addition, there exist sets $\Gamma_{n} \subset \Gamma$ such that $\Gamma=$ $\bigcup_{n \in \mathbb{N}} \Gamma_{n}$ and each $d_{\Gamma_{n}}$ fragments $K$.

Proof. There is a decomposition $\Gamma=\bigcup_{n \in \mathbb{N}} \Gamma_{n}$ such that each $\sigma\left(\Gamma_{n}\right)$ has a bound $\tau_{n}$ in $\left(\mathbb{N}^{\mathbb{N}}, \leq\right)$. We choose $\tau_{n} \in \mathrm{P}$. First, we prove that each $d_{\Gamma_{n}}$ fragments $K$. For every $n \in \mathbb{N}, K$ is fragmented by the map

$$
f_{n}(x, y)=\sup _{\gamma \in \Gamma_{n}} h^{\sigma(\gamma)}\left(\left|x_{\gamma}-y_{\gamma}\right|\right) \leq f(x, y)
$$

and

$$
\begin{aligned}
f_{n}(x, y) & =\sup _{\gamma \in \Gamma_{n}} h^{\sigma(\gamma)}\left(\left|x_{\gamma}-y_{\gamma}\right|\right) \\
& \geq \sup _{\gamma \in \Gamma_{n}} h^{\tau_{n}}\left(\left|x_{\gamma}-y_{\gamma}\right|\right) \\
& =h^{\tau_{n}}\left(\sup _{\gamma \in \Gamma_{n}}\left|x_{\gamma}-y_{\gamma}\right|\right)=h^{\tau_{n}}\left(d_{\Gamma_{n}}(x, y)\right) .
\end{aligned}
$$

Hence, a set of $f_{n}$-diameter less than $1 / \tau_{n}$ in $K$ is a set of $d_{\Gamma_{n}}$-diameter less than $1 / n$ and therefore, since $f_{n}$ fragments $K$, also $d_{\Gamma_{n}}$ fragments $K$.

Consider now the natural projection $p_{n}:[0,1]^{\Gamma} \rightarrow[0,1]^{\Gamma_{n}}$ and $K_{n}=$ $p_{n}(K)$. By Lemma 12 , since $K$ is fragmented by $f_{n}, K_{n}$ is fragmented by

$$
g_{n}(x, y)=\sup _{\gamma \in \Gamma_{n}} h^{\sigma(\gamma)}\left(\left|x_{\gamma}-y_{\gamma}\right|\right),
$$

and hence $K_{n}$ is Radon-Nikodým compact. Moreover, since $\Gamma=\bigcup_{n \in \mathbb{N}} \Gamma_{n}$, $K$ embeds into the product $\prod_{n \in \mathbb{N}} K_{n}$ and the class of Radon-Nikodým compacta is closed under taking countable products and closed subspaces [10], it follows that $K$ is Radon-Nikodým compact. 
Proof of Theorem 3. If the weight of $K$ is less than $\mathbf{b}$, then $K$ can be embedded into a cube $[0,1]^{\Gamma}$ with $|\Gamma|<\mathbf{b}$. Any subset of $\mathbb{N}^{\mathbb{N}}$ of cardinality less than $\mathbf{b}$ is $\sigma$-bounded, so Theorem 3 follows directly from Theorem 10 and Lemma 13.

Proof of Proposition 4. Let $K$ be quasi-Radon-Nikodým compact, suppose $K$ is embedded into some cube $[0,1]^{\Gamma}$ and let $\sigma: \Gamma \rightarrow \mathrm{P}$ be as in Theorem 10. Let $A \subset \mathrm{P}$ be a cofinal subset of $\mathrm{P}$ of cardinality $\mathbf{d}$. For $\alpha \in A$, let

$$
\Gamma_{\alpha}=\{\gamma \in \Gamma: \sigma(\gamma) \leq \alpha\}
$$

let $p_{\alpha}:[0,1]^{\Gamma} \rightarrow[0,1]^{\Gamma_{\alpha}}$ be the natural projection, and let $K_{\alpha}=p_{\alpha}(K)$. Again, since $\Gamma=\bigcup_{\alpha \in A} \Gamma_{\alpha}, K$ embeds into the product $\prod_{\alpha \in A} K_{\alpha}$. By Lemma $12, K_{\alpha}$ is fragmented by

$$
g_{\alpha}(x, y)=\sup _{\gamma \in \Gamma_{\alpha}} h^{\sigma(\gamma)}\left(\left|x_{\gamma}-y_{\gamma}\right|\right) .
$$

The set $\left\{\sigma(\gamma): \gamma \in \Gamma_{\alpha}\right\}$ is bounded, and hence $\sigma$-bounded. Hence, by Lemma $13, K_{\alpha}$ is Radon-Nikodým compact.

We note that from Lemma 13, we obtain something stronger than Theorem 3:

THEOREM 14. For every quasi-Radon-Nikodým compact subset of a cube $[0,1]^{\Gamma}$ with $|\Gamma|<\mathbf{b}$ there is a countable decomposition $\Gamma=\bigcup_{n \in \mathbb{N}} \Gamma_{n}$ such that $d_{\Gamma_{n}}$ fragments $K$ for all $n \in \mathbb{N}$.

A similar result holds also for generalized Cantor cubes (cf. [6, Theorem 3], [2, Theorem 3.6]): If $K$ is a quasi-Radon-Nikodým compact subset of $\{0,1\}^{\Gamma}$, then there is a decomposition $\Gamma=\bigcup_{n \in \mathbb{N}} \Gamma_{n}$ such that $d_{\Gamma_{n}}$ fragments $K$ for all $n \in \mathbb{N}$. We now give an example which shows that this phenomenon does not happen for general cubes, even if the compact $K$ has weight less than $\mathbf{b}$ or is zero-dimensional:

Proposition 15. There exist a set $\Gamma$ of cardinality $\mathbf{b}$ and a compact subset $K$ of $[0,1]^{\Gamma}$ homeomorphic to the metrizable Cantor cube $\{0,1\}^{\mathbb{N}}$ such that for any decomposition $\Gamma=\bigcup_{n \in \mathbb{N}} \Gamma_{n}$ there exists $n \in \mathbb{N}$ such that $d_{\Gamma_{n}}$ does not fragment $K$.

Proof. First, we take a subset $\Gamma$ of $\mathbb{N}^{\mathbb{N}}$ of cardinality b which is not $\sigma$-bounded. We let $A=\left\{\gamma_{n}: \gamma \in \Gamma, n \in \mathbb{N}\right\}$ be the set of all terms of elements of $\Gamma$. We define

$$
K^{\prime}=\left\{x \in\{0,1\}^{\Gamma \times \mathbb{N}}: x_{\gamma, n}=x_{\gamma^{\prime}, n^{\prime}} \text { whenever } \gamma_{n}=\gamma_{n^{\prime}}^{\prime}\right\} .
$$

Observe that $K^{\prime}$ is homeomorphic to $\{0,1\}^{\mathbb{N}}$ : namely, for each $a \in A$ choose some $\gamma^{a}, n^{a} \in \Gamma \times \mathbb{N}$ such that $\gamma_{n^{a}}^{a}=a$; in this case we have a homeomorphism $K^{\prime} \rightarrow\{0,1\}^{A}$ given by $x \mapsto\left(x_{\gamma^{a}, n^{a}}\right)_{a \in A}$. 
Now, we consider the embedding $\phi:\{0,1\}^{\Gamma \times \mathbb{N}} \rightarrow[0,1]^{\Gamma}$ given by

$$
\phi(x)=\left(\sum_{n \in \mathbb{N}}\left(\frac{2}{3}\right)^{n} x_{\gamma, n}\right)_{\gamma \in \Gamma} .
$$

We claim that the space $K=\phi\left(K^{\prime}\right) \subset[0,1]^{\Gamma}$ satisfies the statement. Let $\Gamma=\bigcup_{n \in \mathbb{N}} \Gamma_{n}$ be any countable decomposition of $\Gamma$. Since $\Gamma$ is not $\sigma$-bounded, there is some $n \in \mathbb{N}$ such that $\Gamma_{n}$ is not bounded. For this fixed $n$, since $\Gamma_{n}$ is not bounded, there is some $m \in \mathbb{N}$ such that the set $S=\left\{\gamma_{m}: \gamma \in \Gamma_{n}\right\} \subset A$ is infinite. We consider

$$
K_{0}=\left\{x \in K^{\prime}: x_{\gamma, k}=0 \text { whenever } \gamma_{k} \notin S\right\} \subset K .
$$

By the same arguments as for $K^{\prime}, K_{0}$ is homeomorphic to the Cantor cube $\{0,1\}^{\mathbb{N}}$ by a map $K_{0} \rightarrow\{0,1\}^{S}$ given by $x \mapsto\left(x_{\gamma^{a}, n^{a}}\right)_{a \in S}$. Now, we take two different elements $x, y \in K_{0}$. Then there must exist some $\gamma \in \Gamma_{n}$ such that $x_{\gamma, m} \neq y_{\gamma, m}$, and this implies that $\left|\phi(x)_{\gamma}-\phi(y)_{\gamma}\right| \geq 3^{-m}$ and therefore $d_{\Gamma_{n}}(\phi(x), \phi(y)) \geq 3^{-m}$. This means that any nonempty subset of $\phi\left(K_{0}\right)$ of $d_{\Gamma_{n}}$-diameter less than $3^{-m}$ must be a singleton. If $d_{\Gamma_{n}}$ fragmented $K$, this would imply that $\phi\left(K_{0}\right)$ has an isolated point, which contradicts the fact that it is homeomorphic to $\{0,1\}^{\mathbb{N}}$.

2. Banach spaces of low density character. In this section we find that the cardinal $\mathbf{b}$ is the least possible density character of Banach spaces which are counterexamples to several questions. First, we introduce some notation: If $A$ is a subset of a Banach space $V$, we denote by $d_{A}$ the pseudometric $d_{A}\left(x^{*}, y^{*}\right)=\sup _{x \in A}\left|x^{*}(x)-y^{*}(x)\right|$ on $B_{V^{*}}$. Also, we recall the following definition [5, Definition 1.4.1]:

Definition 16. A nonempty bounded subset $M$ of a Banach space $V$ is called an Asplund set if for each countable set $A \subset M$ the pseudometric space $\left(B_{V^{*}}, d_{A}\right)$ is separable.

By [3, Theorem 2.1], $M$ is an Asplund subset of $V$ if and only if $d_{M}$ fragments $\left(B_{V^{*}}, w^{*}\right)$. Also, by [5, Theorem 1.4.4], a Banach space $V$ is Asplund generated if and only if it is the closed linear span of an Asplund subset.

Proof of Theorem 5. Let $\Gamma$ be a dense subset of the unit ball $B_{V}$ of $V$ of cardinality less than b. Then we have a natural embedding $\left(B_{V^{*}}, w^{*}\right) \subset$ $[-1,1]^{\Gamma}$. Since $\left(B_{V^{*}}, w^{*}\right)$ is quasi-Radon-Nikodým compact, we apply Theorem 14 and we have $\Gamma=\bigcup \Gamma_{n}$ and each $d_{\Gamma_{n}}$ fragments $\left(B_{V^{*}}, w^{*}\right)$. This means that for each $n, \Gamma_{n}$ is an Asplund set, and by [5, Lemma 1.4.3], $M=\bigcup_{n \in \mathbb{N}} \frac{1}{n} \Gamma_{n}$ is an Asplund set as well. Finally, since the closed linear span of $M$ is $V$, by [5, Theorem 1.4.4], $V$ is Asplund generated.

We now recall the concepts that we need for the proof of Theorem 8 . We follow the terminology and notation of [5, Sections 3.1, 4.1]. Let $X$ and $Y$ 
be topological spaces. A map $\phi: X \rightarrow 2^{Y}$ from $X$ to the subsets of $Y$ is said to be an usco if the following conditions hold:

(1) $\phi(x)$ is a compact subset of $Y$ for all $x \in X$.

(2) $\{x: \phi(x) \subset U\}$ is open in $X$, for every open subset $U$ of $Y$.

In this situation, for $A \subset X$ we write $\phi(A)=\bigcup_{x \in A} \phi(x)$.

A completely regular topological space $X$ is said to be $\mathcal{K}$-analytic if there exists an usco $\phi: \mathbb{N}^{\mathbb{N}} \rightarrow 2^{X}$ such that $\phi\left(\mathbb{N}^{\mathbb{N}}\right)=X$. A Banach space is weakly $\mathcal{K}$-analytic if it is a $\mathcal{K}$-analytic space in its weak topology.

We note that if a Banach space $V$ contains a weakly $\sigma$-compact subset $M$ which is dense in the weak topology, then $V$ is WCG. This is because if $M=\bigcup_{n=1}^{\infty} K_{n}$ where $K_{n}$ is a weakly compact set bounded by $c_{n}>0$, then $\{0\} \cup \bigcup\left(1 / n c_{n}\right) K_{n}$ is a weakly compact subset of $V$ whose linear span is (weakly) dense in $V$. Hence, Theorem 8 is deduced from the following:

Proposition 17. If $X$ is a $\mathcal{K}$-analytic topological space which contains a dense subset of cardinality less than $\mathbf{b}$, then $X$ contains a dense $\sigma$-compact subset.

Proof. We have an usco $\phi: \mathbb{N}^{\mathbb{N}} \rightarrow 2^{X}$ with $\phi\left(\mathbb{N}^{\mathbb{N}}\right)=X$ and also a set $\Sigma \subset \mathbb{N}^{\mathbb{N}}$ such that $|\Sigma|<\mathbf{b}$ and $\phi(\Sigma)$ is dense in $X$. Any subset of $\mathbb{N}^{\mathbb{N}}$ of cardinality less than $\mathbf{b}$ is contained in a $\sigma$-compact subset of $\mathbb{N}^{\mathbb{N}}[4$, Theorem 9.1]. Uscos send compact sets to compact sets, so if $\Sigma^{\prime} \supset \Sigma$ is $\sigma$-compact, then $\phi\left(\Sigma^{\prime}\right)$ is a dense $\sigma$-compact subset of $X$.

We recall that a completely regular topological space $X$ is $\mathcal{K}$-countably determined if there exists a subset $\Sigma$ of $\mathbb{N}^{\mathbb{N}}$ and an usco $\phi: \Sigma \rightarrow 2^{X}$ such that $\phi(\Sigma)=X$, and that a Banach space is weakly countably determined if it is $\mathcal{K}$-countably determined in its weak topology. Talagrand [17] has constructed a Banach space which is weakly countably determined but which is not weakly $\mathcal{K}$-analytic. A slight modification of this example gives a similar one with density character $\omega_{1}$. This shows that no analogue of Theorem 8 is possible for weakly countably determined Banach spaces. The change in the example consists in replacing the set $T$ considered in [17, p. 78] by any subset $T^{\prime} \subset T$ of cardinality $\omega_{1}$ such that $\left\{o(X): X \in T^{\prime}\right\}$ is uncountable, and $\mathcal{A}$ by $\mathcal{A}^{\prime}=\left\{A \subset T^{\prime}: A \in \mathcal{A}_{1}\right\}$ (the notations are explained in [17]).

Now, we turn to the fact that the cardinal $\mathbf{b}$ is best possible in Theorems 5 and 8 and their corollaries. We fix a subset $S$ of $\mathbb{N}^{\mathbb{N}}$ of cardinality b which is not $\sigma$-bounded.

Following the exposition of the example of Argyros in [5, Section 1.6] we just replace the space $Y=\overline{\operatorname{span}}\left\{\pi_{\sigma}: \sigma \in \mathbb{N}^{\mathbb{N}}\right\}$ in [5, Theorem 1.6.3] by $Y^{\prime}=\overline{\operatorname{span}}\left\{\pi_{\sigma}: \sigma \in S\right\}$ and we obtain a Banach space of density character b which is a subspace of a WCG space $C(K)$ but which is not Asplund generated. The same arguments in $\left[5\right.$, Section 1.6] hold just with $\mathbb{N}^{\mathbb{N}}$ replaced 
by $S$ where necessary. Only the proof of [5, Lemma 1.6.1] is not good for this case. It must be substituted by the following:

Lemma 18. Let $\Gamma_{n}, n \in \mathbb{N}$, be any subsets of $S$ such that $\bigcup_{n \in \mathbb{N}} \Gamma_{n}=S$. Then there exist $n, m \in \mathbb{N}$ and an infinite set $A \in \mathcal{A}_{m}$ such that $A \subset \Gamma_{n}$.

Here, as in [5, Section 1.6], $\mathcal{A}_{m}$ is the family of all subsets $A \subset \mathbb{N}^{\mathbb{N}}$ such that if $\sigma, \tau \in A$ and $\sigma \neq \tau$, then $\sigma_{i}=\tau_{i}$ if $i \leq m$ and $\sigma_{m+1} \neq \tau_{m+1}$. Also, $\mathcal{A}=\bigcup_{m=1}^{\infty} \mathcal{A}_{m}$.

Proof of Lemma 18. We consider $\Gamma_{i, j}=\left\{\sigma \in \Gamma_{i}: \sigma_{1}=j\right\}, i, j \in \mathbb{N}$. Note that $S=\bigcup_{i, j} \Gamma_{i, j}$. Since $S$ is not $\sigma$-bounded, there exist $n, l$ with $\Gamma_{n, l}$ unbounded. This implies that for some $m$, the set $\left\{\sigma_{m}: \sigma \in \Gamma_{n, l}\right\}$ is infinite. We take the least integer $m$ with this property $(m>1)$. Let $B \subset \Gamma_{n, l}$ be an infinite set such that $\sigma_{m} \neq \sigma_{m}^{\prime}$ for $\sigma, \sigma^{\prime} \in B, \sigma \neq \sigma^{\prime}$. Since all $\sigma_{k}$ with $\sigma \in B, k<m$, lie in a finite set, an infinite set $A \subset B$ can be chosen such that $A \in \mathcal{A}_{m-1}$.

On the other hand, if we follow the proof in [5, Section 4.3] that the Banach space $C(K)$ of Talagrand is weakly $\mathcal{K}$-analytic but not WCG, and we change $K$ in $[5$, p. 76$]$ to $K^{\prime}=\left\{\chi_{A}: A \in \mathcal{A}, A \subset S\right\} \subset\{0,1\}^{S}$ then $C\left(K^{\prime}\right)$ still satisfies this condition and has density character $\mathbf{b}$. Observe that $C\left(K^{\prime}\right)$ is weakly $\mathcal{K}$-analytic because $K^{\prime}$ is a retract of the original $K$. The fact that $C\left(K^{\prime}\right)$ is not WCG (nor even a subspace of a WCG space) follows from [5, Theorem 4.3.2] and Lemma 18 above by the same arguments as in $[5$, p. 78$]$.

3. Countably lower fragmentable compacta. In this section we prove that the concept of quasi-Radon Nikodým compact [2] is equivalent to that of countably lower fragmentable compact [6]. The main result for this class in [6] is that if $K$ is countably lower fragmentable, then so is $\left(B_{C(K)^{*}}, w^{*}\right)$. We note that, with these two facts at hand, together with the fact that if $C(K)$ is Asplund generated, then $K$ is Radon-Nikodým [5, Theorem 1.5.4], Theorem 3 is deduced from Theorem 5 .

We need some notation: if $K$ is a compact space and $A \subset C(K)$ is a bounded set of continuous functions over $K$, we define the pseudometric $d_{A}$ on $K$ as $d_{A}(x, y)=\sup _{f \in A}|f(x)-f(y)|$. If $X$ is a topological space, $d: X \times X \rightarrow \mathbb{R}$ is a map, and $\Delta$ is a positive real number, then $d$ is said to $\Delta$-fragment $X$ if for each subset $L$ of $X$ there is a relative open subset $U$ of $L$ of $d$-diameter less than or equal to $\Delta$.

Definition 19. A compact space $K$ is said to be countably lower fragmentable if there are bounded subsets $\left\{A_{n, p}: n, p \in \mathbb{N}\right\}$ of $C(K)$ such that $C(K)=\bigcup_{n \in \mathbb{N}} A_{n, p}$ for every $p \in \mathbb{N}$, and $K$ is $1 / p$-fragmented by the pseudometric $d_{A_{n, p}}$. 
This is the definition as it appears in [6]. However, the variable $p$ is superfluous in it. If the sets $A_{n, 1}$ exist, it is sufficient to define $A_{n, p}=$ $\left\{(1 / p) f: f \in A_{n, 1}\right\}$.

On the other hand, we recall a concept introduced by Namioka [10]: For a topological space $K$, a set $L \subset K \times K$ is said to be an almost neighborhood of the diagonal if it contains the diagonal $\Delta_{K}=\{(x, x): x \in K\}$ and for every nonempty subset $X$ of $K$ there is a nonempty relative open subset $U$ of $X$ such that $U \times U \subset L$. The use of this was suggested to us by I. Namioka and simplifies our original proof.

THEOREM 20. For a compact subset $K$ of $[0,1]^{\Gamma}$ the following are equivalent:

(1) $K$ is quasi-Radon-Nikodým compact.

(2) $K$ is countably lower fragmentable.

(3) There are subsets $\Gamma_{n, p}, n, p \in \mathbb{N}$, of $\Gamma$ such that $K$ is $1 / p$-fragmented by $d_{\Gamma_{n, p}}$ for every $n, p \in \mathbb{N}$.

Proof. Suppose $K$ is quasi-Radon-Nikodým compact and let $\phi$ be a lower semicontinuous quasi-metric which fragments $K$. Then we just define

$$
\begin{aligned}
A_{n, p}=\{f \in C(K):|f(x)-f(y)|<1 / p \text { whenever } & \phi(x, y) \leq 1 / n\} \\
& \cap\left\{f:\|f\|_{\infty} \leq n\right\} .
\end{aligned}
$$

Clearly, $K$ is $1 / p$-fragmented by $d_{A_{n, p}}$ because any subset of $K$ of $\phi$-diameter less than $1 / n$ has $d_{A_{n, p}}$-diameter less than $1 / p$, and we know that $\phi$ fragments $K$. On the other hand, for a fixed $p \in \mathbb{N}$, in order to prove that $C(K)=\bigcup_{n \in \mathbb{N}} A_{n, p}$, observe that, if $f \in C(K)$, then

$$
C_{n}=\{(x, y) \in K \times K:|f(x)-f(y)| \geq 1 / p \text { and } \phi(x, y) \leq 1 / n\}
$$

is a decreasing sequence of compact subsets of $K \times K$ with empty intersection, so there is some $n>\|f\|_{\infty}$ such that $C_{n}$ is empty, and then $f \in A_{n, p}$.

That (2) implies (3) is evident, just take $\Gamma_{n, p}=A_{n, p} \cap \Gamma$, where $A_{n, p}$, $n, p \in \mathbb{N}$, are the sets in the definition of countable lower fragmentability.

Suppose (3). For every $n, p \in \mathbb{N}$, since $K$ is $1 / p$-fragmented by $d_{A_{n, p}}$, the set $C_{n, p}=\left\{(x, y) \in K \times K: d_{\Gamma_{n, p}}(x, y) \leq 1 / p\right\}$ is an almost neighborhood of the diagonal which, in addition, is closed. On the other hand, for each $n, p \in \mathbb{N},(x, y) \in C_{n, p}$ if and only if $\left|x_{\gamma}-y_{\gamma}\right| \leq 1 / p$ for all $\gamma \in \Gamma_{n, p}$ so that

$$
\bigcap_{n, p \in \mathbb{N}} C_{n, p}=\bigcap_{p \in \mathbb{N}}\left\{(x, y):\left|x_{\gamma}-y_{\gamma}\right| \leq 1 / p \text { for all } \gamma \in \bigcup_{n \in \mathbb{N}} \Gamma_{n, p}=\Gamma\right\}=\Delta_{K} \text {. }
$$

Now, $K$ is quasi-Radon-Nikodým by virtue of [11, Theorem 1], which states that $K$ is quasi-Radon-Nikodým compact if and only if there is a countable family of closed almost neighborhoods of the diagonal whose intersection is the diagonal $\Delta_{K}$. 
A. Avilés

\section{References}

[1] A. V. Arkhangel'skiı̌ (ed.), General Topology. II, Encyclopaedia Math. Sci. 50, Springer, Berlin, 1996.

[2] A. D. Arvanitakis, Some remarks on Radon-Nikodým compact spaces, Fund. Math. 172 (2002), 41-60.

[3] B. Cascales, I. Namioka, and J. Orihuela, The Lindelöf property in Banach spaces, Studia Math. 154 (2003), 165-192.

[4] E. K. van Douwen, The integers and topology, in: Handbook of Set-Theoretic Topology, North-Holland, Amsterdam, 1984, 111-167.

[5] M. J. Fabian, Gâteaux Differentiability of Convex Functions and Topology. Weak Asplund Spaces, CMS Ser. Monogr. Adv. Texts, Wiley, New York, 1997.

[6] M. Fabian, M. Heisler, and E. Matoušková, Remarks on continuous images of Radon-Nikodým compacta, Comment. Math. Univ. Carolin. 39 (1998), 59-69.

[7] D. H. Fremlin, Consequences of Martin's Axiom, Cambridge Tracts in Math. 84, Cambridge Univ. Press, Cambridge, 1984.

[8] S. P. Gul'ko, On properties of subsets of $\Sigma$-products, Soviet Math. Dokl. 18 (1977), 1438-1442.

[9] S. Mercourakis and E. Stamati, A new class of weakly $\mathcal{K}$-analytic Banach spaces, Mathematika, to appear.

[10] I. Namioka, Radon-Nikodým compact spaces and fragmentability, ibid. 34 (1987), $258-281$.

[11] —, On generalizations of Radon-Nikodým compact spaces, Topology Proc. 26 (2002), $741-750$.

[12] J. Orihuela, W. Schachermayer, and M. Valdivia, Every Radon-Nikodým Corson compact space is Eberlein compact, Studia Math. 98 (1991), 157-174.

[13] O. I. Reynov, On a class of Hausdorff compacts and GSG Banach spaces, ibid. 71 (1981/82), 294-300.

[14] H. P. Rosenthal, The heredity problem for weakly compactly generated Banach spaces, Compositio Math. 28 (1974), 83-111.

[15] C. Stegall, Spaces of Lipschitz functions on Banach spaces, in: Functional Analysis (Essen, 1991), Lecture Notes in Pure and Appl. Math. 150, Dekker, New York, 1994, $265-278$.

[16] M. Talagrand, Espaces de Banach faiblement $\mathcal{K}$-analytiques, Ann. of Math. (2) 110 (1979), 407-438.

[17] —, A new countably determined Banach space, Israel J. Math. 47 (1984), 75-80.

Departamento de Matemáticas

Universidad de Murcia

30100 Espinardo (Murcia), Spain

E-mail: avileslo@um.es

Received December 23, 2003

Revised version May 4, 2004 